\title{
Time to Recovery from Severe Acute Malnutrition and Its Predictors among Admitted Children Aged 6-59 Months at the Therapeutic Feeding Center of Pawi General Hospital, Northwest Ethiopia: A Retrospective Follow-Up Study
}

\author{
Amare Wondim (D), Bethelihem Tigabu, and Mengistu Mekonnen Kelkay $(1)$ \\ Department of Pediatric and Child Health Nursing, School of Nursing, College of Medicine and Health Sciences, University of Gondar, \\ Gondar, Ethiopia \\ Correspondence should be addressed to Amare Wondim; amarewondim556@gmail.com
}

Received 12 August 2019; Revised 1 January 2020; Accepted 1 February 2020; Published 11 March 2020

Academic Editor: Namik Y. Ozbek

Copyright (c) 2020 Amare Wondim et al. This is an open access article distributed under the Creative Commons Attribution License, which permits unrestricted use, distribution, and reproduction in any medium, provided the original work is properly cited.

\begin{abstract}
Background. Ethiopia is one of the countries in sub-Saharan Africa with the highest rates of severe acute malnutrition. Early recovery is a performance indicator for severe acute malnourished children for the therapeutic feeding. Despite the available interventions to tackle nutritional problems, there is scarce information on time to recovery and its determinants among children with SAM in Ethiopia. Objective. The study is aimed at assessing time to recovery from severe acute malnutrition and its predictors among admitted children aged 6-59 months at the therapeutic feeding center of Pawi General Hospital, northwest Ethiopia, from January 2013 to December 2017. Methods. An institution-based retrospective follow-up study was conducted among 398 children aged 6-59 months. The data were collected by using data extraction sheet. The data were cleaned and entered using EpiData version 4.2.0.0 and exported to Stata version 14 statistical software for further analysis. Kaplan-Meier survival curve was used to estimate median nutritional recovery time after initiation of inpatient treatment, and log-rank test was used to compare time to recovery between groups. The Cox proportional regression model was used to identify the predictors of recovery time. Adjusted hazard rate with its 95\% CI was reported to show strength of relationship. Results. The recovery rate was 5.3 per 100 person-day observations, and the median recovery time was 14 days (95\% CI: 13-15). The lower chance of early recovery was found among children who were not fully vaccinated (AHR: 0.73 (95\% CI: 0.56, 0.96)), while high chance of recovery was found among children who had no anemia (AHR: 1.66 (95\% CI: 1.23, 2.23)), TB (AHR: 2.03 (95\% CI: $1.11,3.71)$ ), and malaria infection (AHR: $1.54(95 \%$ CI: 1.09, 2.17)) at admission. Conclusion and Recommendation. The overall nutritional recovery rate was below the accepted minimum standard. Children not fully vaccinated and children without malaria, anemia, and TB comorbidities at admission had a higher chance of recovering early from severe acute malnutrition. Hence, treating comorbidities is vital for prompt nutritional recovery.
\end{abstract}

\section{Introduction}

Severe acute malnutrition (SAM) is defined as very low weight for height $<70 \%$, visible severe wasting, or the presence of nutritional edema. In children aged 6-59 months, mid-upper arm circumference (MUAC) less than $115 \mathrm{~mm}$ is also indicative of severe acute malnutrition $[1,2]$.

Globally, 17 million children are living with severe acute malnutrition; 1 to 2 million die every year; 25 to $30 \%$ of the deaths are in many poor countries [2]. Most of the children with SAM live in South Asia and sub-Saharan Africa. Furthermore, it is the most common cause of hospital admissions, accounting for $20 \%$ of pediatric hospital admissions in Ethiopia [3].

Ethiopia is still one of the countries with the highest rates of severe acute malnutrition despite a small decline from $12 \%$ to $10 \%$ over the last 15 years. The prevalence of severe acute malnutrition among under five-year-old children in the 


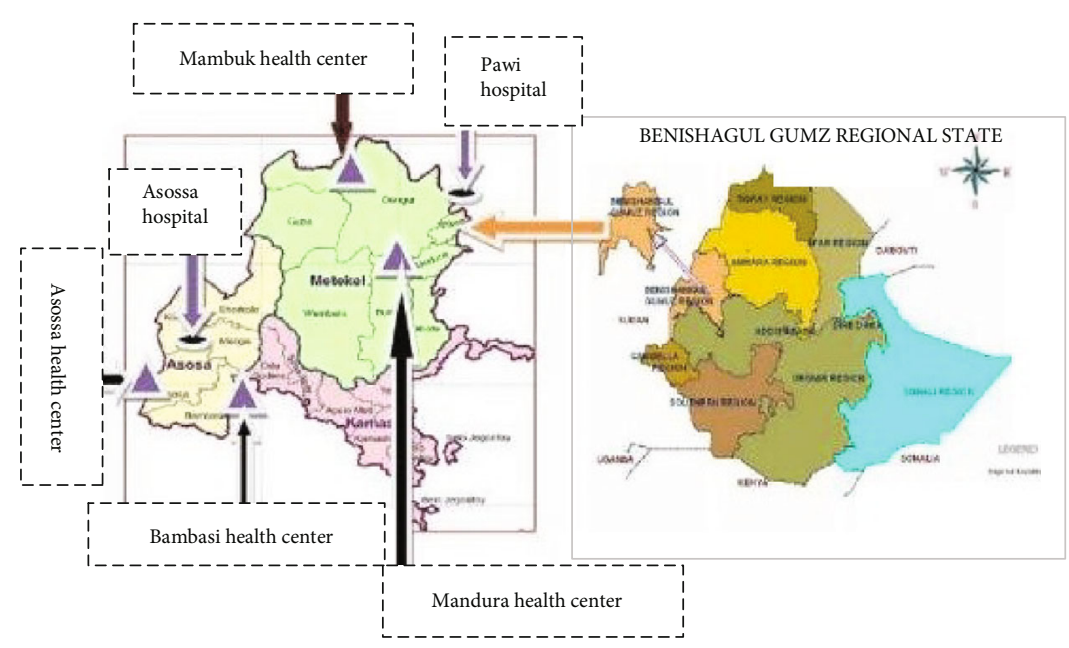

Figure 1: Sketch map of the study setting adapted from a previous study [19].

country is estimated at 2.9\%; in the study where the setting was Benishangul-Gumuz region, it was 3.1\% [4]. The annual cost associated with SAM is estimated to be US $\$ 262.62$ per child in Ethiopia [3]. Besides, delayed discharge from severe acute malnutrition increases the number of children to be treated, medicine cost, and human resource demand, thus imposing economic burden on resource-poor settings $[5,6]$. Furthermore, if left untreated, it might result in the impairment of physical, intellectual, social, and adaptive behavior $[7,8]$.

Studies reported inconsistencies in the rate of recovery from SAM which ranges from 22.1 to $95.36 \%$. In Ethiopia, the rate of recovery is known to be below the accepted international minimum standard of 75\%. For instance, Mekele, Bahir Dar, and Gondar reported 22.1\% (9), 58.4\% [9], and $68.5 \%$ [10], respectively. Likewise, studies conducted in Ethiopia on the time to recovery from SAM ranged from 14 to 26 days [9, 11-18]. Shorter recovery time shows an acceptable performance of the treatment and caring process. Recovery time is affected by medical comorbidities like anemia, malaria, dehydration, hypoglycemia, HIV, TB, hypothermia, and lack of child full vaccination. Though these predictors are assumed to delay the time to recovery, some investigators show conflicting results in listing the possible causes of prolonged recovery time.

Although Ethiopia adapted the WHO SAM guideline and has been working for Sustainable Development Goals 2025 under its nutritional strategy goal in order to end hunger, achieve food security, and improve nutrition, only limited information exists regarding the outcome of the SAM treatment provided at the therapeutic feeding center. Ethiopia Demographic and Health Survey (EDHS) reported that the Benishangul-Gumuz region had a high prevalence rate. However, no studies have been conducted in the study area on nutritional recovery. Therefore, this study is aimed at determining the time to recovery from severe acute malnutrition and its predictors among children aged 6-59 months admitted to Pawi General Hospital, northwest Ethiopia.

\section{Methods and Materials}

2.1. Study Design, Setting, and Period. A hospital-based retrospective follow-up study was conducted on SAM cases enrolled at the hospital from 2013 to 2017. Data was collected from March to April 2018. The study was conducted in the Benishangul-Gumuz regional state, Pawi General Hospital. Pawi is found in the Metekel zone of the BenishangulGumuz regional state, $570 \mathrm{~km}$ away from Addis Ababa, the capital of Ethiopia. The study site is geographically located at $11.009^{\prime} \mathrm{N}$ latitude and $36.003^{\prime} \mathrm{E}$ longitude and at an altitude of 1050 meters above sea level. The mean annual rainfall and maximum temperature of the area are $1555.1 \mathrm{~mm}$ and $32^{\circ} \mathrm{C}$, respectively. The hospital has different departments that provide specialized services in outpatient, inpatient, and operation theatre departments that serve an estimated 300,000 people in the region, have 200 beds, and have around 1000 inpatients admitted per month on average. The average patient flow to the therapeutic feeding center was 300/year and $25 /$ month for 37 beds (Figure 1 ).

All children aged 6-59 months and treated for severe acute malnutrition at the therapeutic feeding center of Pawi General Hospital enrolled from January 2013 to December 2017 were included in this study. Children in affected areas were screened for signs of SAM and diagnosed based on anthropometric measurement and examination of the feet for bilateral pitting edema. Children who fulfill the criteria for admission were admitted to the TFC, for example, if the $\mathrm{W} / \mathrm{H}$ was $<70 \%$ of the median WHO growth standard, if the MUAC was found to be less than $11 \mathrm{~cm}$, or if the children had bilateral pedal edema. The data were obtained by reviewing records from the inpatient therapeutic feeding registration book and individual follow-up chart.

2.2. Sample Size Determination. From a total of 420 children with SAM admitted and treated at the therapeutic feeding center (TFC) of the hospital from January 2013 to December 2017, 398 were selected. After evaluation, 22 of them were excluded as they did not have admission and discharge date. 
Sample size was determined using Stata version 14; for survival functions, a Cox model based on a retrospective follow-up study conducted in Bahir Dar Felege Hiwot Referral Hospital was used to asses predictors of nutritional recovery time and predictors with SAM having HIV $(\mathrm{CHR}=2.35)$, malaria $(\mathrm{CHR}=1.904)$, and TB $(\mathrm{CHR}=2.49)$ [20] (Table 1$)$.

2.3. Data Collection Tool and Procedure. Data were extracted for one month by trained nurses using data extraction sheet prepared in the English language after checking all available data on the severe acute malnutrition inpatient registration book and medical patient charts. The data extraction sheet crosschecked with preestablished known source study variables. Lists of charts of study participants during the study period were taken from the card room, and the charts were coded. Finally, charts which have complete baseline data were selected and variables were recorded.

The data extraction form was developed after reviewing relevant literature from the standard treatment protocol for the management of severe acute malnutrition, SAM registration log book, and monitoring multicharts. It includes child sociodemographic characteristics (age, sex, and residence), anthropometric measurements (height, weight, and MUAC), comorbidities, and treatment outcomes of the child.

2.4. Variables of the Study. The dependent variable of the study is time to recovery from SAM (i.e., the event of interest is recovery and the response variable is rate of recovery). The independent variables considered are child sex, age, residence, comorbidities (TB, HIV, cough (pneumonia), anemia, malaria, diarrhea, vomiting, hypoglycemia, dehydration, hypothermia, septic shock, vaccination status, and dermatitis (skin lesion)), type of malnutrition (marasmus, kwashiorkor, and marasmus-kwashiorkor), and routine drugs (folic acid supplement, amoxicillin, and deworming medication).

2.5. Operational Definitions. Recovered: weight for height greater than or equal to $85 \%$ of the median WHO growth chart reference, absence of bilateral pitting edema, and no medical complication [21]

Time to recovery: time to recovery was obtained by calculating the difference (in days) from the start of treatment until the child recovered [13]

Censored: those who were referred, were nonresponders, were defaulted, and died

Defaulters: those who leave the treatment before the child is cured/lost with unknown status, when the patient discontinued before recovery [20]

2.6. Data Management and Analysis. Data were entered into EpiData version 4.2.0.0 after checking for completeness, and then the data was cleaned and exported to Stata version 14 for analysis. Descriptive analyses for continuous and categorical data describing the cohort characteristics at baseline and during follow-up were made.

Schoenfeld residual analysis (global test) was used to show the Cox proportional hazard model assumption valid at $P$ value $=0.2536$. Predictors of time to recovery were identified using bivariable and multivariable Cox proportional hazard models (CPHM). All independent variables that had
TABLE 1

\begin{tabular}{lccccc}
\hline Predictors & Power & Recovery & Withdrawal & CHR & Sample size \\
\hline HIV & $90 \%$ & 0.519 & 0.481 & 2.35 & 215 \\
TB & $90 \%$ & 0.519 & 0.481 & 2.49 & 188 \\
Malaria & $90 \%$ & 0.519 & 0.481 & 1.904 & 379 \\
\hline
\end{tabular}

$P$ value less than 0.25 in the bivariable model were considered candidate variables for the multivariable model. The output of the multivariable CPHM is presented using adjusted hazard ratios (AHR) with the respective 95\% confidence intervals (CI). Kaplan-Meier survival estimator and log-rank tests were used to estimate median recovery time during the treatment period and to compare time to recovery between groups, respectively. The Cox-Snell plot was used to check the overall model fitness. Multicollinearity was checked using the variance inflation factor.

\section{Results}

3.1. Sociodemographic and Baseline Characteristics. Out of 398 children in the cohort, $56.03 \%$ were males and $70.35 \%$ were from rural areas. The median age was 19 (IQR: 12-32) months. Considering clinical features, $49.75 \%$ and $47.24 \%$ were marasmus and kwashiorkor, respectively. For marasmic children, the median MUAC was $10(9.4-10.5) \mathrm{cm}$ and the median length/height and weight of the children were 74 (IQR: $66-81) \mathrm{cm}$ and 6.8 (IQR: $5.5-8.5) \mathrm{kg}$, respectively (Table 2).

3.2. Routine Medication and Treatment. Out of the 398 children, the majority (97.7) received amoxicillin and $85.9 \%$ received deworming medication, more than half $(57.3 \%)$ took vitamin A supplement, and almost all (99.5\%) received folic acid supplement. However, only $10.8 \%$ of children received IV fluid (Table 3 ).

3.3. Treatment Outcomes, Nutritional Recovery Rate, and Time of Recovery of Children with Severe Acute Malnutrition. From 398 children, 262 (65.8\%, 95\% CI: 60.9$70.5 \%)$ recovered, 108 (27.1\%) were cured, and 154 (38.7\%) transferred out to OTP (Figure 2).

From those, $65.3 \%$ recovered within 28 days with the median time to recovery of 14 day (95\% CI: 13-15). Incidence of recovery was 5.3 (95\% CI: 4.7-6.0) per 100 person-day observations. Out of these, $33.2 \%$ recovered within the second week, followed by $82(20.6 \%)$ within the third week (Figure 3 ).

However, the recovery rate and time were varied with predictors, including anemia and tuberculosis (Table 4).

3.4. Predictors of Time to Recovery from Severe Acute Malnutrition. The multivariable Cox regression analysis showed that malaria, TB, anemia, breast feeding status, and vaccination status were found to be the predictors of time to recovery from SAM. Those who had no malaria at baseline had increased chance of recovery from SAM by 1.54 (AHR: 1.54 (95\% CI: 1.09, 2.17)) compared to those who had malaria.

At admission, those who had vaccination status not appropriate for the age had 27\% (AHR: 0.73 (95\% CI: 0.55, 
TABLE 2: Sociodemographic and baseline characteristics of 6-59month-old children with severe acute malnutrition in the therapeutic feeding center at Pawi General Hospital, northwest Ethiopia (2013-2017) $(n=398)$.

\begin{tabular}{|c|c|c|}
\hline Variable & Character & $\begin{array}{c}\text { Frequency } \\
(\%)\end{array}$ \\
\hline \multirow{2}{*}{ Sex } & Male & $223(56.0)$ \\
\hline & Female & $175(44.0)$ \\
\hline \multirow{2}{*}{ Residence } & Rural & $281(70.6)$ \\
\hline & Urban & $117(29.4)$ \\
\hline \multirow{5}{*}{ Age of the child (months) } & $6-11$ & $97(24.4)$ \\
\hline & $12-23$ & $116(29.2)$ \\
\hline & $24-35$ & $96(24.0)$ \\
\hline & $36-47$ & $42(10.6)$ \\
\hline & $48-59$ & $47(11.8)$ \\
\hline \multirow{3}{*}{ SAM diagnosis type } & Marasmus & $198(49.8)$ \\
\hline & Kwashiorkor & $188(47.2)$ \\
\hline & $\begin{array}{l}\text { Marasmus- } \\
\text { kwashiorkor }\end{array}$ & $12(3.0)$ \\
\hline \multirow{4}{*}{ Vaccination status } & Appropriate for age & $166(41.7)$ \\
\hline & Not appropriate for age & $147(36.9)$ \\
\hline & Not documented & $15(3.8)$ \\
\hline & Not vaccinated & $70(17.6)$ \\
\hline \multirow{2}{*}{ Hypoglycemia } & Absent & $78(95.0)$ \\
\hline & Present & $20(5.0)$ \\
\hline \multirow{2}{*}{ Dermatitis } & Absent & $348(87.4)$ \\
\hline & Present & $50(12.6)$ \\
\hline \multirow{2}{*}{ Dehydration } & No & $190(47.7)$ \\
\hline & Yes & $208(52.3)$ \\
\hline \multirow{2}{*}{ Vomiting } & Absent & $261(65.6)$ \\
\hline & Present & $137(34.4)$ \\
\hline \multirow{2}{*}{ Diarrhea } & Absent & $191(48.0)$ \\
\hline & Present & $207(52.0)$ \\
\hline \multirow{2}{*}{ HIV } & Yes & $1(0.3)$ \\
\hline & No & $397(99.7)$ \\
\hline \multirow{2}{*}{ Septic shock } & Yes & $18(4.5)$ \\
\hline & No & $380(95.5)$ \\
\hline \multirow{2}{*}{ Malaria } & Yes & $76(19.15)$ \\
\hline & No & $322(80.9)$ \\
\hline \multirow{2}{*}{ Anemia } & Yes & $137(34.4)$ \\
\hline & No & $261(65.6)$ \\
\hline \multirow{2}{*}{ Pneumonia } & Yes & $195(49.0)$ \\
\hline & No & $203(51.0)$ \\
\hline \multirow{2}{*}{ TB } & Yes & $27(6.8)$ \\
\hline & No & $371(93.2)$ \\
\hline \multirow{2}{*}{ Hypothermia } & Yes & $11(2.8)$ \\
\hline & No & $387(97.2)$ \\
\hline
\end{tabular}

TABLE 3: Routine medication and supplementation for 6-59month-old children with severe acute malnutrition in the therapeutic feeding center of Pawi General Hospital, northwest Ethiopia (2013-2017) $(n=398)$.

\begin{tabular}{lc}
\hline Characteristics & Frequency $(\%)$ \\
\hline Amoxicillin & $389(97.7)$ \\
Yes & $9(2.3)$ \\
No & \\
Folic acid & $396(99.5)$ \\
Yes & $2(0.5 \%)$ \\
No & \\
Deworming & $342(85.9)$ \\
Yes & $56(14.1)$ \\
No & \\
Vitamin A & $228(57.3)$ \\
Yes & $170(42.7)$ \\
No & \\
NG tube feeding & $123(30.9)$ \\
Yes & $275(69.1)$ \\
No & \\
IV fluid & $43(10.8)$ \\
Yes & $355(89.2)$ \\
No & \\
Breast feeding status & $385(96.7)$ \\
Exclusive breast feeding & \\
Mixed & $277(69.6)$ \\
Blood transfusion & $121(30.4)$ \\
Yes & \\
No & \\
Antimalarial drug & \\
Nes & \\
\hline & \\
No & \\
\hline
\end{tabular}

0.95)) less chance of recovery than their counterparts. Children admitted for SAM with no anemia had 1.66 (AHR: $1.66(95 \%$ CI: $1.23,2.23)$ ) times more chance of recovery compared to those who had anemia at baseline.

Children without TB infection at admission were 2.03 (AHR: 2.03 (95\% CI: 1.11, 3.71)) times more likely to recover from SAM compared to those with TB infection at baseline. Children who had mixed feeding were less likely to recover as compared to those who had exclusive breast feeding (AHR: 0.59 (95\% CI: 0.44, 0.81)) (Table 5).

\section{Discussion}

In this study, the recovery rate was 65.8 (95\% CI: $60 \%$, $70.5 \%)$, which was below the minimum accepted international standard of $75 \%$ [22]. It is lower than the finding of studies conducted in various areas of Ethiopia, for example, $77.9 \%$ in Debre Markos and Finote Selam [12], 83\% in Woliso St. Luke Hospital [13], 87\% in Jimma Specialized Referral Hospital [14], and 95.36\% in Shebedino [6]. This might be due to differences in human resources and 


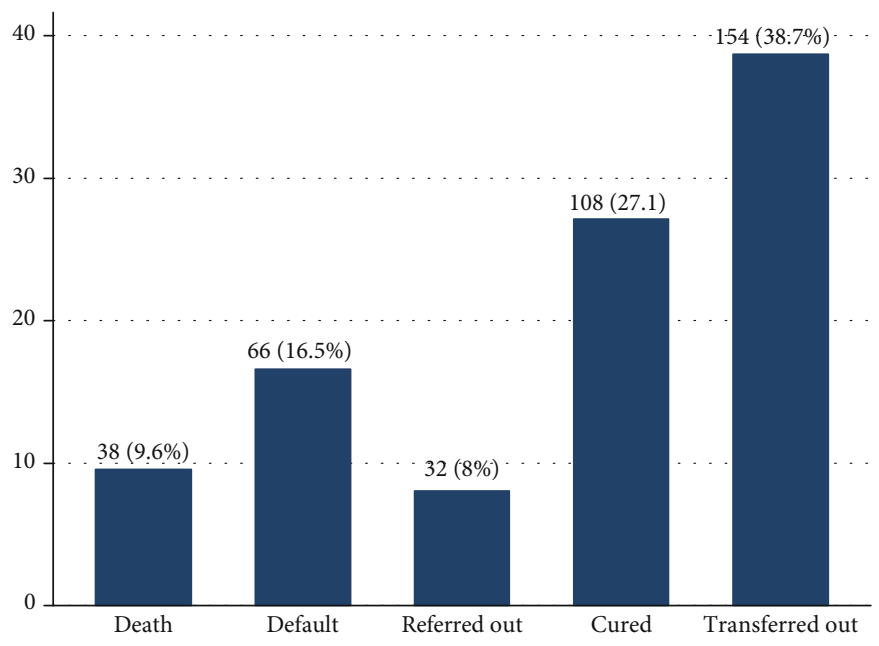

Figure 2: Treatment outcomes of 6-59-month-old children admitted for severe acute malnutrition in the therapeutic feeding center at Pawi General Hospital, northwest Ethiopia, from 2013 to 2017 ( $n=398)$.

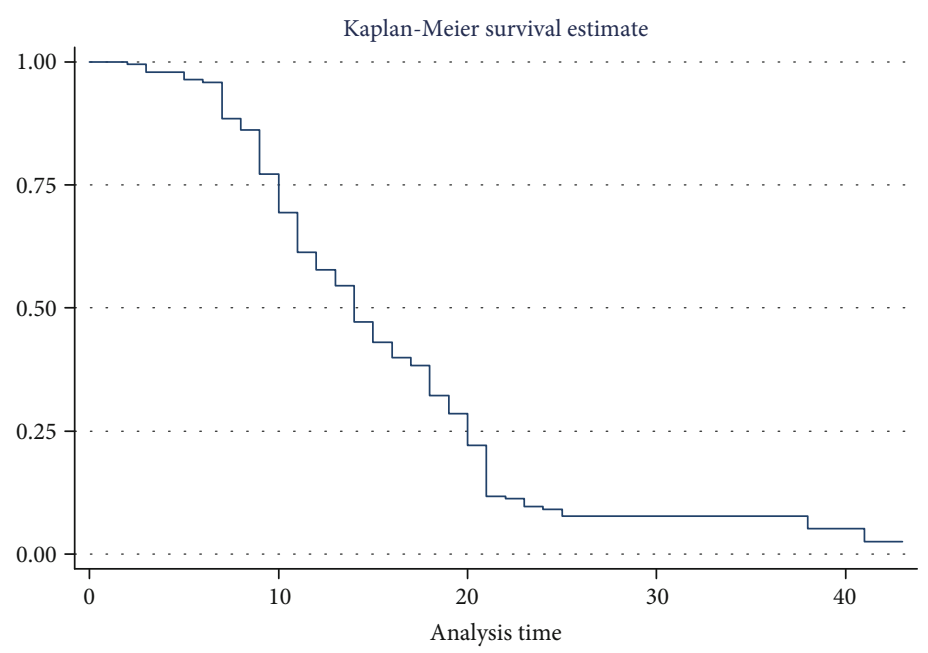

FIGURE 3: Overall median recovery time of the entire cohort in the therapeutic feeding center at Pawi General Hospital, northwest Ethiopia, from 2013 to 2017.

TABLE 4: Recovery time and rate from SAM by predictors of among children with SAM managed at Pawi General Hospital from 2013 to 2017.

\begin{tabular}{|c|c|c|c|c|c|}
\hline Variable & Characteristics & Subjects & Recovery rate (per 100 person-days) & Median (IQR) in days & $95 \% \mathrm{CI}$ \\
\hline \multirow[t]{3}{*}{ Anemia } & Yes & 137 & 4.2 & $19(11,21)$ & $19(16,20)$ \\
\hline & No & 261 & 6.0 & $12(9,18)$ & $12(11,14)$ \\
\hline & Total & 398 & 5.3 & $14(10,20)$ & $14(13,15)$ \\
\hline \multirow[t]{3}{*}{ Vaccination status } & Appropriate & 166 & 6.5 & $13(9,19)$ & $13(11,14)$ \\
\hline & Not appropriate & 232 & 4.4 & $15(10,21)$ & $15(14,18)$ \\
\hline & Total & 398 & 5.3 & $14(10,20)$ & $14(13,15)$ \\
\hline \multirow[t]{3}{*}{$\mathrm{TB}$} & Yes & 27 & 3.5 & $21(12,23)$ & $21(12,23)$ \\
\hline & No & 371 & 5.5 & $14(10,20)$ & $14(13,15)$ \\
\hline & Total & 398 & 5.3 & $14(10,20)$ & $14(13,15)$ \\
\hline \multirow[t]{3}{*}{ Malaria } & Yes & 76 & 4.1 & $19(14,21)$ & $19(18,21)$ \\
\hline & No & 322 & 5.7 & $13(9,19)$ & $13(11,14)$ \\
\hline & Total & 398 & 5.3 & $14(10,20)$ & $14(13,15)$ \\
\hline
\end{tabular}


TABle 5: Predictors of recovery time from severe acute malnutrition among 6-59-month-old children admitted in the therapeutic feeding center of Pawi General Hospital, northwest Ethiopia (2013-2017).

\begin{tabular}{|c|c|c|c|c|c|}
\hline Characteristics & Recovered & Censored & CHR (95\% CI) & $P$ value & AHR (95\% CI) \\
\hline \multicolumn{6}{|l|}{ Residence } \\
\hline Rural & 174 & 106 & 1 & & 1 \\
\hline Urban & 88 & 30 & $1.35(1.05,1.75)$ & 0.0139 & $1.23(0.93,1.61)$ \\
\hline \multicolumn{6}{|l|}{ Age } \\
\hline $6-23$ & 131 & 82 & 1 & & 1 \\
\hline 24-59 & 131 & 54 & $1.19(0.80,1.30)$ & 0.200 & $1.2(0.92,1.57)$ \\
\hline \multicolumn{6}{|l|}{ Malaria } \\
\hline Yes & 46 & 29 & 1 & & 1 \\
\hline No & 216 & 107 & $1.90(1.38,2.62)$ & 0.001 & $1.54(1.09,2.17)$ \\
\hline \multicolumn{6}{|l|}{ Diarrhea } \\
\hline Absent & 128 & 63 & 1 & & 1 \\
\hline Present & 134 & 73 & $0.81(0.64,1.04)$ & 0.072 & $1.17(0.88,1.55)$ \\
\hline \multicolumn{6}{|l|}{$\mathrm{TB}$} \\
\hline Yes & 13 & 14 & 1 & & 1 \\
\hline No & 249 & 122 & $2.05(1.17,3.60)$ & 0.0053 & $2.03(1.11,3.71)$ \\
\hline \multicolumn{6}{|l|}{ Pneumonia } \\
\hline Absent & 147 & 55 & 1 & & 1 \\
\hline Present & 115 & 82 & $0.76(0.59,0.97)$ & 0.018 & $1.02(0.77,1.34)$ \\
\hline \multicolumn{6}{|l|}{ Dermatitis } \\
\hline Absent & 222 & 125 & 1 & & 1 \\
\hline Present & 40 & 11 & $1.77(1.25,2.49)$ & 0.139 & $1.36(0.93,2.00)$ \\
\hline \multicolumn{6}{|l|}{ Hypoglycemia } \\
\hline Absent & 257 & 120 & 1 & & 1 \\
\hline Present & 5 & 16 & $0.39(0.14,1.05)$ & 0.144 & $0.41(0.15,1.12)$ \\
\hline \multicolumn{6}{|l|}{ Vaccination status } \\
\hline Appropriate for age & 138 & 28 & 1 & & 1 \\
\hline Not appropriate for age & 108 & 124 & $0.68(0.53,0.87)$ & 0.0009 & $0.73(0.55,0.95)$ \\
\hline \multicolumn{6}{|l|}{ Anemia } \\
\hline Yes & 79 & 58 & 1 & & 1 \\
\hline No & 183 & 78 & $1.88(1.44,2.47)$ & 0.001 & $1.66(1.23,2.23)$ \\
\hline \multicolumn{6}{|l|}{ Vomiting } \\
\hline Yes & 177 & 84 & $0.73(0.56,0.95)$ & 0.122 & $0.85(0.64,1.13)$ \\
\hline No & 85 & 52 & 1 & & 1 \\
\hline \multicolumn{6}{|l|}{ Breast feeding status } \\
\hline Exclusive & 200 & 77 & 1 & & 1 \\
\hline Mixed & 62 & 59 & $0.55(0.41,0.73)$ & 0.001 & $0.59(0.44,0.81)$ \\
\hline \multicolumn{6}{|l|}{ ReSoMal } \\
\hline Yes & 163 & 74 & 1 & & 1 \\
\hline No & 99 & 62 & $1.38(1.07,1.78)$ & 0.114 & $1.27(0.94,1.69)$ \\
\hline
\end{tabular}

The global proportionality assumption test $(P$ value $=0.2536)$.

appropriateness of vaccinations for age. However, it is higher than the 22.1\% noted in Mekele [11] and 58. 4\% noted in Bahir Dar Felege Hiwot Referral Hospital [9].

The median recovery time of 14 days $(95 \%$ CI: 13,15$)$ was within the acceptable minimum international standard set for therapeutic feeding centers which is less than 28 days [22]. The finding is in line with those of studies conducted in Woliso and Gedeo zones [13, 23], but it was lower than that in Tigray (21days) [11], Yirgalem (18.6 days) [24], Jimma (21 days) [14], Bahir Dar (16 days) [20], and Karat referral hospitals (26 days) [25]. The discrepancy could be due to differences in the study setting as the latter studies were conducted in referral and specialized hospitals where children with the most severe SAM cases are referred.

In this study, children who had no anemia at admission were more likely to recover early compared to their 
counterparts. This was congruent with study reports in Bahir Dar Felege Hiwot Referral Hospital and Fasha and Karat $[9,26]$. The reason might be that malnourished children are more likely to have micronutrient deficiency particularly iron deficiency anemia and that intervention was not initiated as early as possible as iron damages cell membranes and makes infections worse. Besides, other medical complications also exacerbate time to recovery $[27,28]$. Similar to other studies reported elsewhere $[9,24,29]$, children without TB infection at admission were 2.03 times more likely to recover from SAM as compared to those with TB infection at baseline. This could be explained by the fact that the presence of malnutrition facilitates the progress of $\mathrm{TB}$ infection to an active disease due to its immunosuppressive effects. Moreover, tuberculosis creates a potentially lethal cycle of worsening illness and deteriorating nutritional status, ultimately resulting in a longer recovery time [30]. This finding revealed that severely malnourished children with no malaria infection recovered early compared to children with malaria infection. This is supported by studies conducted in Niger, Amazon, and Rwanda [25, 31, 32]. The reason might be that malaria impairs gluconeogenesis and increases energy consumption, induces malabsorption, predisposes one to loss of immunity, and results in inability respond to standard treatments $[32,33]$.

Severely malnourished children who had vaccination status not appropriate for the age were $27 \%$ less likely to recover earlier than their counterparts. The finding is supported by results in developing countries [34]. The impact of severe acute malnutrition will be worsened as innate and adaptive immune dysfunction leads to immune dysregulation $[34,35]$.

Children who had history of mixed feeding were $41 \%$ less likely to recover earlier than children who had history of exclusive breast feeding. The finding is in line with a study reported by World Health Organization previously [36] and a finding from Dire Dawa, eastern Ethiopia [37]. Mixed feeding may impose additional pathogen and complicate the management of children with SAM.

\section{Strengths and Limitations}

The strength of the study was the use of the advanced statistical analysis model. Further, this study is one among few studies in Ethiopia particularly novice for the study setting that were conducted to assess treatment outcomes and factors affecting time to recovery among 6-59-month-old children with SAM admitted at the Pawi General Hospital stabilization center. Due to the nature of the retrospective and secondary data, incomplete records were seen in some variables. We were unable to explore other sociodemographic characteristics, such as maternal and paternal educational status.

\section{Conclusion}

In the study area, the overall recovery rate was low. Children whose vaccination status was not appropriate for age and who had malaria, anemia, TB comorbidities, and history of mixed feeding at admission were predictors associated with time to recovery from severe acute malnutrition. Thus, emphasis should be given to prompt and timely management of malaria, anemia, and TB and more importantly to universal vaccination coverage appropriate for age.

$\begin{array}{ll}\text { Abbreviations } \\ \text { AHR: } & \text { Adjusted hazard ratios } \\ \text { CPHM: } & \text { Cox proportional hazard models } \\ \text { CHR: } & \text { Crude hazard ratio } \\ \text { CI: } & \text { Confidence intervals } \\ \text { GDP: } & \text { Gross domestic product } \\ \text { EDHS: } & \text { Ethiopia Demographic and Health Survey } \\ \text { HIV: } & \text { Human immunodeficiency virus } \\ \text { IQR: } & \text { Interquartile range } \\ \text { IV: } & \text { Intravenous } \\ \text { MUAC: } & \text { Mid-upper arm circumference } \\ \text { NG: } & \text { Nasogastric tube } \\ \text { SAM: } & \text { Severe acute malnutrition } \\ \text { SD: } & \text { Standard deviation } \\ \text { TB: } & \text { Tuberculosis } \\ \text { TFC: } & \text { Therapeutic feeding center } \\ \text { WHO: } & \text { World Health Organization } \\ \chi^{2}: & \text { Chi-square. }\end{array}$

\section{Data Availability}

The datasets used and/or analyzed and in which conclusions are drawn during the current study are included in the manuscript.

\section{Ethical Approval}

Ethical clearance was obtained from the Ethical Review Committee of School of Nursing on behalf of the Institutional Review Board of University of Gondar. Information in the data abstraction tool was nonidentifying and anonymous. The files of entered data in the software and the final result of this study were tied with passwords. Confidentiality and privacy of the information were kept safe throughout the whole process of the research work.

\section{Conflicts of Interest}

The authors declare that they have no competing interests.

\section{Authors' Contributions}

BT and MM conceived, designed, and organized the whole procedure of this article production and performed data analysis and interpretation of findings. AW and BT performed data analysis, interpretation of findings, and coaching and mentoring and prepared the manuscript. BT, $\mathrm{AW}$, and $\mathrm{MM}$ performed data analysis, interpretation of findings, and coaching and mentoring and equally prepared the manuscript. All authors read and approved the final manuscript. 


\section{Acknowledgments}

The authors' appreciation goes to Pawi General Hospital administrative bodies, data collectors, and charts room worker for their cooperation and permission to conduct the study. This study was funded by the Amhara Regional State Health Bureau and Debretabore Health Science College.

\section{References}

[1] Federal democratic Republic of Ethiopia and Ministry of Health, Training course on the management of severe acute malnutrition, Ministry of Health, Addis Ababa, 2nd edition, 2013.

[2] World Health Organization and UNICEF, Community-based management of severe acute malnutrition: a joint statement by the World Health Organization, the World Food Programme, the United Nations System Standing Committee on Nutrition and the United Nations Children's Fund, 2007.

[3] African Union, The social and economic impacts of child under nutrition in Ethiopia, summary report, 2015.

[4] Central Statistical Agency (CSA) [Ethiopia] and ICF, Ethiopia Demographic and Health Survey 2016, CSA and ICF, Addis Ababa, Ethiopia, and Rockvile, Maryland, USA, 2016.

[5] WHO and UNICEF, WHO child growth standards and the identification of severe acute malnutrition in infants and children, World Health Organization, Geneva: Swizerland, 2009.

[6] A. Tekeste, M. Wondafrash, G. Azene, and K. Deribe, "Cost effectiveness of community-based and in-patient therapeutic feeding programs to treat severe acute malnutrition in Ethiopia," Cost Effectiveness and Resource Allocation, vol. 10, no. 1, p. 4, 2012.

[7] D. M. Gordon, S. Frenning, H. R. Draper, and M. Kokeb, "Prevalence and burden of diseases presenting to a general pediatrics ward in Gondar, Ethiopia," Journal of Tropical Pediatrics, vol. 59, no. 5, pp. 350-357, 2013.

[8] World Health Organization and Unicef, WHO child growth standards and the identification of severe acute malnutrition in infants and children, World Health Organization and the United Nations Children's Fund, 2009.

[9] H. D. Desyibelew, A. Fekadu, and H. Woldie, "Recovery rate and associated factors of children age 6 to 59 months admitted with severe acute malnutrition at inpatient unit of Bahir Dar Felege Hiwot referral hospital therapeutic feeding unite, Northwest Ethiopia," PLoS One, vol. 12, no. 2, article e0171020, 2017.

[10] A. Abeje, T. Gudayu, Y. Malefia, and B. Befftu, "Analysis of hospital records on treatment outcome of severe acute malnutrition: the case of Gondar University tertiary hospital," Pediatrics and Therapeutics, vol. 6, p. 283, 2016.

[11] M. G. Tirore, T. M. Atey, and H. B. Mezgebe, "Survival status and factors associated with treatment outcome of severely malnourished children admitted to Ayder referral hospital: a cross-sectional study," BMC Nutrition, vol. 3, no. 1, 2017.

[12] G. Mekuria, T. Derese, and G. Hailu, "Treatment outcome and associated factors of severe acute malnutrition among 6-59 months old children in Debre Markos and Finote Selam hospitals, Northwest Ethiopia: a retrospective cohort study," BMC Nutrition, vol. 3, no. 1, 2017.

[13] A. Banbeta, D. Seyoum, T. Belachew, B. Birlie, and Y. Getachew, "Modeling time-to-cure from severe acute mal- nutrition: application of various parametric frailty models," Archives of Public Health, vol. 73, no. 1, 2015.

[14] C. Misganaw, M. Mesfin, M. Tesfaye, and A. Derese, "Retrospective study on outcome of in-patient treatment of severe acute malnutrition in Jimma University specialized hospital from September 2011-September 2012," Journal of Diagnostics, vol. 1, no. 1, pp. 18-27, 2014.

[15] T. Girum, M. Kote, B. Tariku, and H. Bekele, "Survival status and predictors of mortality among severely acute malnourished children $<5$ years of age admitted to stabilization centers in Gedeo zone: a retrospective cohort study," Therapeutics and Clinical Risk Management, vol. 13, pp. 101-110, 2017.

[16] M. Saaka, S. M. Osman, A. Amponsem et al., "Treatment outcome of severe acute malnutrition cases at the tamale teaching hospital," Journal of Nutrition and Metabolism, vol. 2015, Article ID 641784, 8 pages, 2015.

[17] E. Teferi, M. Lera, S. Sita, Z. Bogale, D. G. Datiko, and M. A. Yassin, "Treatment outcome of children with severe acute malnutrition admitted to therapeutic feeding centers in Southern Region of Ethiopia," Ethiopian Journal of Health Development, vol. 24, no. 3, 2010.

[18] E. M. A. Tette, E. K. Sifah, and E. T. Nartey, "Factors affecting malnutrition in children and the uptake of interventions to prevent the condition," BMC Pediatrics, vol. 15, no. 1, 2015.

[19] H. Disassa, K. Tafess, A. Worku, and G. Ameni, "A preliminary study on molecular characterization of mycobacterium tuberculosis in Benishangul Gumuz region, Western Ethiopia," Microbiology Research Journal International, vol. 10, no. 6, pp. 1-10, 2015.

[20] D. T. Asres, R. P. C. J. Prasad, and T. A. Ayele, "Recovery time and associated factors of severe acute malnutrition among children in Bahir Dar city, Northwest Ethiopia: an institution based retrospective cohort study," BMC Nutrition, vol. 4, no. $1,2018$.

[21] S. Chamois, M. Golden, and Y. Grellety, Ethiopia protocol for the management of severe acute malnutrition, Ethiopian Federal Ministry of Health, Addis Ababa, 2007.

[22] T. Van Zutphen, Sphere Project-Humanitarian Charter and Minimum Standards in Humanitarian Response, Practical Action Publishing, Warwickshire, UK, 2011.

[23] T. Girum, M. Kote, B. Tariku, and H. Bekele, "Survival status and predictors of mortality among severely acute malnourished children \&lt; 5 years of age admitted to stabilization centers in Gedeo zone: a retrospective cohort study," Therapeutics and Clinical Risk Management, vol. 13, pp. 101-110, 2017.

[24] A. Kabeta and G. Bekele, "Factors associated with treatment outcomes of under-five children with severe acute malnutrition admitted to therapeutic feeding unit of Yirgalem Hospital," Clinics Mother Child Health, vol. 14, no. 261, p. 2, 2017.

[25] F. Kateera, C. M. Ingabire, E. Hakizimana et al., "Malaria, anaemia and under-nutrition: three frequently co-existing conditions among preschool children in rural Rwanda," Malaria Journal, vol. 14, no. 1, 2015.

[26] D. Y. Gebremichael, "Predictors of nutritional recovery time and survival status among children with severe acute malnutrition who have been managed in therapeutic feeding centers, southern Ethiopia: retrospective cohort study," BMC Public Health, vol. 15, no. 1, 2015.

[27] N. Thakur, J. Chandra, H. Pemde, and V. Singh, "Anemia in severe acute malnutrition," Nutrition, vol. 30, no. 4, pp. 440 $442,2014$. 
[28] FMOH, Guideline for managment of severe acute malnutrition, Federal Ministry of Health, 2013.

[29] A. Schoonees, M. Lombard, A. Musekiwa, E. Nel, J. Volmink, and Cochrane Developmental, Psychosocial and Learning Problems Group, "Ready-to-use therapeutic food for homebased treatment of severe acute malnutrition in children from six months to five years of age," Cochrane Database of Systematic Reviews, vol. 2019, 2013.

[30] S. O. H. Kanan and M. O. Swar, "Prevalence and outcome of severe malnutrition in children less than five-year-old in Omdurman Paediatric Hospital, Sudan," Sudanese Journal of Paediatrics, vol. 16, no. 1, pp. 23-30, 2016.

[31] Z. W. Adama, C. W. Ella, M. D. Bengaly et al., "Determinants of mortality in children under five years of age with severe acute malnutrition admitted to the Yalgado Ouédraogo Teaching Hospital (Burkina Faso)," International Journal of Child Health and Nutrition, vol. 5, no. 1, pp. 1-9, 2016.

[32] M. A. A. Alexandre, S. G. Benzecry, A. M. Siqueira et al., "The association between nutritional status and malaria in children from a rural community in the Amazonian region: a longitudinal study," PLoS Neglected Tropical Diseases, vol. 9, no. 4, article e0003743, 2015.

[33] A. H. Shankar, "Nutritional modulation of malaria morbidity and mortality," The Journal of Infectious Diseases, vol. 182, Supplement 1, pp. S37-S53, 2000.

[34] A. J. Prendergast, "Malnutrition and vaccination in developing countries," Philosophical Transactions of the Royal Society B: Biological Sciences, vol. 370, no. 1671, 2015.

[35] A. Ray, N. K. Sinha, S. Maiti, S. K. Roy, and K. Maiti, "Reciprocity between partial immunization and malnutrition significantly impairs health of preschool children," Indian Journal of Maternal and Child Health, vol. 15, no. 1, p. 9, 2013.

[36] R. G. Feachem and M. A. Koblinsky, "Interventions for the control of diarrhoeal diseases among young children: promotion of breast-feeding," Bulletin of the World Health Organization, vol. 62, no. 2, pp. 271-291, 1984.

[37] B. Atnafe, K. T. Roba, and T. Dingeta, “Time of recovery and associated factors of children with severe acute malnutrition treated at outpatient therapeutic feeding program in Dire Dawa, Eastern Ethiopia," PLoS One, vol. 14, no. 6, article e0217344, 2019. 